\title{
Accuracy of $d^{\prime}$ and $A^{\prime}$ as estimates of sensitivity
}

\author{
WAYNE DONALDSON \\ University of New Brunswick, Fredericton, New Brunswick, Canada
}

\begin{abstract}
In signal detection theory, sensitivity can be indexed as either the distance between distributions or the area under the isosensitivity curve. The statistic $d^{\prime}$ is usually identified as a distance measure of sensitivity, while $A^{\prime}$ is an estimate of the area. This article considers both statistics as estimates. As such, they can be evaluated against actual distances and areas calculated under specific assumptions about the variances of the distributions. The analysis shows that $d^{\prime}$ is a better estimate of distance than $A^{\prime}$ is of area only under the assumption that the variances of the hypothetical distributions are equal (i.e., the slope of the $z$-coordinate ROC is 1.0 ). When the slope takes on values other than $1.0, A^{\prime}$ is generally a more accurate estimate of area than $d^{\prime}$ is of distance.
\end{abstract}

Yes-no recognition memory tasks require observers to decide whether or not items were previously presented to them. Data collected in such tasks are reduced to two numbers. The hit rate $(\mathrm{H})$ is the proportion of previously presented items correctly recognized as old items. The false-alarm rate $(F)$ is the proportion of new items incorrectly identified as old items. These two numbers are affected by the ability of the observer to remember (sensitivity or discrimination) and by the willingness of the observer to say so (response bias). Numerous approaches try to derive from $\mathrm{H}$ and $\mathrm{F}$ independent descriptive statistics of sensitivity and bias.

This recognition memory task is only one example of what Macmillan and Creelman (1991) call correspondence experiments. The simplest correspondence experiments establish two stimulus classes. Observers are made aware of the two classes and are then asked to decide which of the two classes is the source of a current stimulus. All measures ${ }^{1}$ of sensitivity and bias are based on models of underlying processes. Macmillan and Creelman present these models and discuss about a dozen different measures of sensitivity and at least as many measures of response bias. Concentrating on the recognition memory task, Snodgrass and Corwin (1988) evaluated four models that generate four measures of sensitivity and seven of response bias. Theoretically, and when applied to data, some measures from some models outperform others, and the interested reader should consult the original articles (but see Donaldson, 1992).

Measures from signal detection theory performed well in both the Macmillan and Creelman (1991) and the Snodgrass and Corwin (1988) analyses. The success of the approach is further reflected in the frequency with which $d^{\prime}$ is the sensitivity measure of choice in the litera-

\footnotetext{
This work was completed while the author was on sabbatical leave at the University of California at Santa Cruz and was supported by a research grant from the University of New Brunswick. Correspondence should be addressed to W. Donaldson, Psychology Department, University of New Brunswick, Fredericton, New Brunswick, Canada E3B 6E4.
}

ture. This statistic indexes sensitivity as the distance between the means of the distributions associated with the two stimulus classes. An alternate choice as an index of sensitivity would be the area under the isosensitivity (ROC) curve, which Green and Moses (1966) demonstrated to have a number of useful characteristics. $A^{\prime}$ is an estimate of that area and is used quite often, although only occasionally with an explanation as to why (e.g., Warm, Dember, Murphy, \& Dittmar, 1992). This article evaluates the accuracy of the two estimates, $d^{\prime}$ and $A^{\prime}$, against actual distances and areas calculated under various assumptions.

This evaluation of $d^{\prime}$ and $A^{\prime}$ was prompted by one major concern. There is agreement on the first requirement of a good measure of sensitivity. Macmillan and Creelman (1991) say that "a good sensitivity measure should be invariant when factors other than sensitivity change" (p. 13). Snodgrass and Corwin $(1988$, p. 35) concur, requiring that "the discrimination index be invariant across explicit manipulations of bias." Evaluations then begin by assuming that "the standard deviation of the old distribution equals the standard deviation of the new distribution" (Snodgrass \& Corwin, 1988, p. 36). In other words, evaluations occur only under conditions where the sensitivity estimate $d^{\prime}$ is by definition independent of criterion changes. This article explores the adequacy of the measures without being restricted to the equal variance assumption.

Macmillan and Creelman (1991) do not ignore the issue. They point out that the $(\mathrm{H}, \mathrm{F})$ pairs $(.83, .31)$ and $(.96, .62)$ both yield a $d^{\prime}$ of 1.45 . The adequacy of these estimates rests on the assumption that both points fall on the $z$-coordinate isosensitivity curve with a slope of 1.0 (i.e., the equal variance assumption). "But if the true ROCs have slopes of, say, 0.5 , the second point reflects much greater sensitivity than the first" (Macmillan \& Creelman, 1991, p. 83). Indeed, assuming a slope of 0.5 , the more appropriate measure of sensitivity, $d_{\mathrm{a}}$ (Macmillan \& Creelman, 1991, p. 70), produces values of 1.52 and 2.02 , respectively. Thus, using $d^{\prime}$ of 1.45 as a mea- 


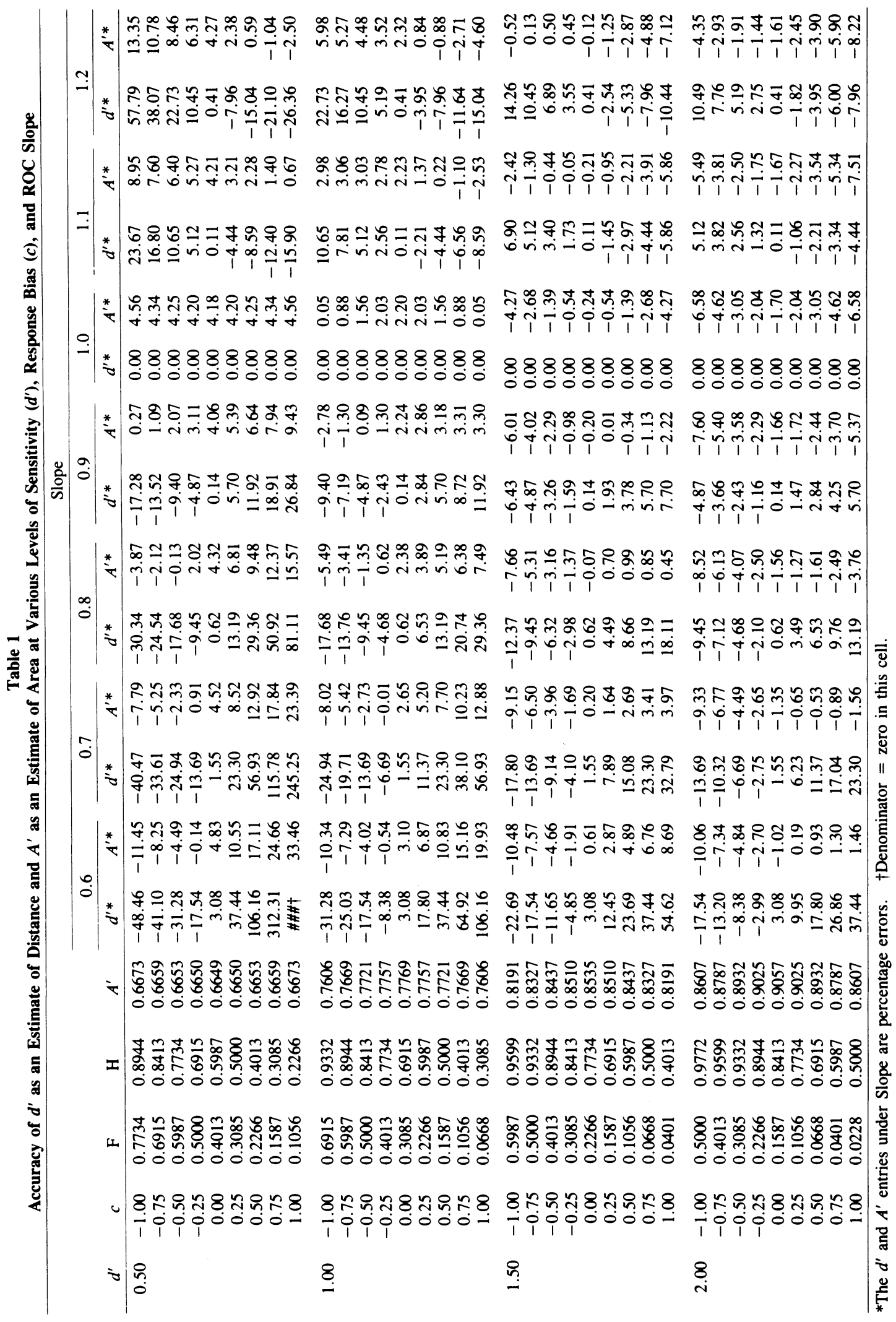


sure would underestimate sensitivity by $4.6 \%$ and $28.2 \%$, respectively. In comparison, using Grier's (1971) formula, the $A^{\prime}$ measures for the two pairs are .8450 and .8122. The actual area under an ROC curve can be derived from $d_{\mathrm{a}}$ and is called $A_{\mathrm{z}}$ by Swets and Pickett (1982). The $A_{\mathrm{z}}$ value for an ROC curve with a $d^{\prime}$ of 1.45 and a slope of $1.00\left(d^{\prime}\right.$ equals $d_{\mathrm{a}}$ when slope is 1.00$)$ is .8474 . With slopes of 0.5 through the two pairs, the actual areas are .8588 and .9234 , respectively. The use of $A^{\prime}$ underestimates these values by $1.6 \%$ and $12.0 \%$. Thus, in these examples, $A^{\prime}$ more accurately estimates area than $d^{\prime}$ does distance. This article systematically explores such comparisons over a range of values of sensitivity, criterion, and slope of the isosensitivity function.

The best way to understand how the exploration was conducted is to work through Table 1 . The four sections of the table represent different levels of sensitivity. To generate the first part of Table 1 , an ROC curve with a $d^{\prime}$ of 0.50 and a $z$-coordinate slope of 1.0 was constructed. ${ }^{2}$ Points were then taken from that curve at criterion $(c)$ values between -1.00 and +1.00 in steps of 0.25 . While this measure of criterion can range up to \pm 2.33 (Macmillan \& Creelman, 1991), the range chosen provides great latitude in response bias. The table shows that with $d^{\prime}$ at 0.50 , a conservative bias as extreme as +1.00 occurs only when the $\mathrm{H}$ drops below 0.23 , and a liberal bias of -1.00 requires an $F$ over 0.77 . The $\mathrm{F}$ and $\mathrm{H}$ values that correspond to the selected criterion values are shown on the third and fourth columns of the table. The value of $A^{\prime}$ associated with each $(\mathrm{H}, \mathrm{F})$ pair of values is shown in the fifth column. At this point, each $(H, F)$ pair in the table was treated as if it were from a Yes-No experiment. The first $(\mathrm{H}, \mathrm{F})$ pair in the table is $(.8944, .7734)$. Suppose that pair actually fell on an ROC curve with a slope of 0.6 instead of 1.0 . The values of $d_{\mathrm{a}}$ and $A_{\mathrm{z}}$ were calculated for a curve with that slope passing through that point. The calculations yield a $d_{\mathrm{a}}$ of 0.9701 and an $A_{\mathrm{z}}$ of 0.7536 . Thus, reporting a $d^{\prime}$ of 0.50 for this $(\mathrm{H}, \mathrm{F})$ pair underestimates $d_{\mathrm{a}}$ by $48.46 \%\left[\left(d^{\prime}-\right.\right.$ $\left.d_{\mathrm{a}}\right) / d_{\mathrm{a}}$ ], and reporting an $A^{\prime}$ of 0.6673 underestimates $A_{\mathrm{z}}$ by $11.45 \%\left[\left(A^{\prime}-A_{z}\right) / A_{z}\right]$. These are the two percentages reported in the first row as $d^{\prime}$ error and $A^{\prime}$ error for a slope of 0.6. Going across the row, the same procedure was repeated when the slope was assumed to take on the other values indicated. Error values were similarly calculated for the other selected points from the original ROC curve. The table then shows the results of the same analysis, beginning with unit slope ROC curves with a $d^{\prime}$ of $1.0,1.5$, and 2.0 .

Three observations seem warranted. First, if one knows or is willing to assume that the ROC curve underlying an $(\mathrm{H}, \mathrm{F})$ pair has a slope of 1.0 , then $d^{\prime}$ shows less error as an estimate of distance than does $A^{\prime}$ of area. Indeed, under such an assumption, there is no error associated with the use of $d^{\prime}$ since $d^{\prime}$ equals $d_{\mathrm{a}}$. $A^{\prime}$ is not error free with respect to $A_{\mathrm{z}}$ in that situation, but the amount of error is not large, exceeding $5 \%$ only with strongly biased performance ( $c$ values of \pm 1.00$)$ at high $\left(d^{\prime}=2.0\right)$ sensitivity. Second, if the $(H, F)$ pair shows unbiased per- formance ( $c=0$, meaning $\mathrm{H}=1-\mathrm{F}$ ), then $d^{\prime}$ tends to be more accurate than $A^{\prime}$, although at no point in the table does the error in either measure reach $\pm 5 \%$. Third, when a slope of 1.0 cannot be assumed and performance shows some bias, $A^{\prime}$ is clearly a more accurate estimate than is $d^{\prime}$. Of the 192 such comparisons in Table $1, A^{\prime}$ is a more accurate estimate of area than $d^{\prime}$ is of distance in 174 of them. And the differences in accuracy between $d^{\prime}$ and $A^{\prime}$ are often not small differences. While $A^{\prime}$ is seldom inaccurate by more than $15 \%$ ( 8 times in 252 calculations), the $d^{\prime}$ estimate error exceeds that value in $67 \mathrm{cal}-$ culations or almost one third of the time when the slope is not 1.0 .

Two main conclusions need to be drawn from this analysis. The first is that both $d^{\prime}$ and $A^{\prime}$ are estimates of something else. Too often the impression is left that $d^{\prime}$ is a measure of sensitivity, while $A^{\prime}$ is but an estimate of a possible measure of sensitivity. Both are estimates. It then becomes a useful exercise to explore the accuracy of those estimates under different sets of assumptions. The most critical assumption concerns the slope of the theoretical isosensitivity function. In general, $A^{\prime}$ is more accurate than $d^{\prime}$, unless performance is unbiased or can be assumed to lie on an ROC curve with a $z$-coordinate slope of 1.0. It should also be noted that these evaluations have been based on the assumption that the underlying hypothetical distributions are Gaussian, central to the analysis of sensitivity as distance but not to that based on area under the ROC curve (Green \& Moses, 1966). The second point is one that has been made many times before. Any reduction of uncertainty about the slope of the isosensitivity curve increases confidence about measures of sensitivity. Collecting confidence ratings reduces that uncertainty. As Macmillan and Creelman (1991, p. 85) point out, "the user of detection theory who does not collect ratings is at risk." The analysis presented here may at least allow such users to assess the degree of risk.

\section{REFERENCES}

DonALDSON, W. (1992). Measuring recognition memory. Journal of Experimental Psychology: General, 121, 275-277.

GreEN, D. M., \& Moses, F. L. (1966). On the equivalence of two recognition measures of short-term memory. Psychological Bulletin, 66 , 228-234.

GRIER, J. B. (1971). Nonparametric indexes for sensitivity and bias: Computing formulas. Psychological Bulletin, 75, 424-429.

Macmillan, N. A., \& Creelman, C. D. (1991). Detection theory: A user's guide. Cambridge: Cambridge University Press.

SNodgrass, J. G., \& CoRWIN, J. (1988). Pragmatics of measuring recognition memory: Applications to dementia and amnesia. Journal of Experimental Psychology: General, 117, 34-50.

Swets, J. A., \& PicketT, R. M. (1982). Evaluation of diagnostic systems: Methods from signal detection theory. New York: Academic Press.

Warm, J. S., Dember, W. N., Murphy, A. Z., \& Dittmar, M. L. (1992). Sensing and decision-making components of the signalregularity effect in vigilance performance. Bulletin of the Psychonomic Society, 30, 297-300. 


\section{NOTES}

1. The term measure will continue to be used even though the premise is that both $d^{\prime}$ and $A^{\prime}$ must be thought of as estimates. Macmillan and Creelman (1991) use the terms measure, index, and statistic interchangeably, but measure is the dominant term.
2. It should be pointed out that constructing the isosensitivity curves with these characteristics biases the analysis against the area measures. The linearity of the $z$-coordinate slope is derived from distributions that are assumed to be Gaussian, and area measures are not limited by that assumption (Green \& Moses, 1966).

(Manuscript received February 1, 1993.) 